\title{
Salud mental positiva en estudiantes del Centro Pre- Universitario de la Universidad Nacional Mayor de San Marcos
}

\author{
Positive mental health in students of the Pre-University Center of the \\ Universidad Nacional Mayor de San Marcos
}
Carlos Velázquez ${ }^{1 *}$, William Montgomery ${ }^{2}$, Alejandro Dioses ${ }^{3}$, Ricardo Pomalaya $^{4}$, Kevin Egúsquiza ${ }^{5}$, Victoria Cavero ${ }^{6}$, Angelika Pizarro, Ofelia Borja $^{8}$, David Duymovich ${ }^{9}$, Giuliana Meza ${ }^{10}$, Dongas Vázquez ${ }^{11}$
Universidad Nacional Mayor de San Marcos

\author{
Natalia Velázquez ${ }^{12}$ \\ Profesional Independiente \\ Susana Cabrera ${ }^{13}$ \\ Universidad César Vallejo
}

\footnotetext{
*Autor para correspondencia

1. Facultad de Psicología, Universidad Nacional Mayor de San Marcos.

Email: cvelasquezc@unmsm.edu.pe

2. Facultad de Psicología, Universidad Nacional Mayor de San Marcos.

Email: wmontgomeryu@unmsm.edu.pe

3. Facultad de Psicología, Universidad Nacional Mayor de San Marcos. Email: adiosesc@unmsm.edu.pe

4. Facultad de Psicología, Universidad Nacional Mayor de San Marcos.

Email: rpomalayav@unmsm-edu.pe.

5. Facultad de Psicología, Universidad Nacional Mayor de San Marcos. Email: kev9nhev@gmail.com

6. Facultad de Psicología, Universidad Nacional Mayor de San Marcos.

Email: victoriamcavero@gmail.com

7. Facultad de Psicología, Universidad Nacional Mayor de San Marcos. Email: angelika93@hotmail.com

8. Facultad de Psicología, Universidad Nacional Mayor de San Marcos.

Email: ofelia.borja@unmsm,edu,pe

9. Facultad de Psicología, Universidad Nacional Mayor de San Marcos.

Email: david_duymovich64g,ail.com

10. Facultad de Psicología, Universidad Nacional Mayor de San Marcos.

Email: gluliana.meza@hotmail.com

11. Facultad de Psicología, Universidad Nacional Mayor de San Marcos.

Email: caritaeangel_0195@hotmail,com

12. Profesional independiente. Email: natalia_ewn@hotmail.com

13. Universidad César Vallejo. Email: susanahay@hotmail.com
} 
Carlos Velázquez, William Montgomery, Alejandro Dioses, Ricardo Pomalaya, Kevin Egúsquiza, Victoria Cavero, Angelika Pizarro, Ofelia Borja, David Duymovich, Giuliana Meza, Dongas Vázquez, Natalia Velázquez y Susana Cabrera

\section{Resumen}

El presente estudio tiene como objetivo la identificación de la Salud Mental Positiva en los alumnos del Centro Pre-universitario de la Universidad Nacional Mayor de San Marcos, para lo cual se seleccionó una muestra accidental de 938 alumnos, donde 436 son del sexo masculino y 502 del sexo femenino. Se adaptó la Escala de Evaluación Mental Positiva (EESMP), obteniéndose un índice de validez de .649 y una confiabilidad entre .637 a .791. Los resultados indican la existen de diferencias en función al sexo en los factores F2, F3 y F5. En cuanto al turno en el que estudian se observa diferencias en F1, F2, F4 y F5; en cuanto a la Institución Educativa de donde provienen no existen diferencias; en el Área profesional donde postulan existen diferencias en los F1, F3 y F4; en el género de pertenencia no existen diferencias, y la correlación entre los factores muestra relaciones significativas positivas y negativas entre bajas y moderadas.

Palabras clave: Salud Mental, salud mental positiva, estudiantes preuniversitarios, áreas profesionales.

\section{Abstract}

The present study aims at the identification of Positive Mental Health in students of the PreUniversity Center of the Universidad Nacional Mayor de San Marcos for which an accidental sample of 938 students of which 436 are male and 502 are female_Adapting Positive Mental Evaluation Scale (EESMP), obtaining a validity between 268 to .649 and a reliability between, 637 to 791 The results indicate that there are differences as a function of sex in factors F2, F3 and F5 As for the shift in which they study, differences are observed in F1, F2, F4 and F5. As far as the Educational Institution from which they come, there are no differences In the Professional Ares where they postulate there are differences in F1, F3 and F4; Sex there are no differences and the correlation between the factors show significant posítive and negative relationships between low. and moderate.

Keywords: Mental health, positive mental health, pre-university students, professional areas.

Conseguir éxito académico y culminar satisfactoriamente el ciclo de formación universitaria depende, en gran medida, de la orientación que la institución universitaria le brinde al estudiante, incidiendo en el grado de adaptación psicológica que aquel consiga en el desempeño de sus exigentes tareas. Cuando dicho grado de adaptación no alcanza el punto preciso, es común encontrar que durante el transcurso de los tres primeros semestres dentro de la institución universitaria se presente un elevado índice o ratio de deserción que puede deberse a diversos factores, entre los que se encuentran en primerísimo lugar problemas económicos (Ramirez, 2009; Plascencia, 2011), pero también desorientación en relación con la carrera, escaso rendimiento, dificultades familiares e incluso embarazo adolescente.

Según las investigaciones sobre problemas como estos, la regla es que los eventos estresantes, en general, incrementan las respuestas de descontrol emotivo y por tanto reducen el nivel de ajuste (Kim, Conger, Elder \& Lorenz; citados por Halo y Mahecha, 2005; p. 200). A su vez, como fue establecido por el modelo transaccional de Lazarus \& Folkman (1986), la experiencia estresante tiene que ver con el mecanismo en que se plasman las transacciones entre la persona y el entorno, las cuales dependen del impacto sobre el sujeto y sus evaluaciones del 
evento ambiental estresor ${ }^{13}$, como por los recursos personales, sociales o culturales de que dispone la persona para enfrentar la situación estresante.

En el plano de la incidencia las estadísticas al respectó de la deserción universit0 son escasas, ya que, como señalaban Marín, Infante y Troyano (2000): "Si bien la literatura en torno al rendimiento o fracaso escolar es sumamente amplia en alumnos no universitarios, la aplicación de esta temática en estudios superiores pasee escasa tradición" (p. 506); y esa realidad no ha cambiado mucho, aunque Inquietud por investigarla es creciente, Así, los datos aportados por la Organización de Cooperación Económica y el Desarrollo (OECD, 2016) indican que las cifras de deserción en los principales países desarrollados están aumentando.

Por ejemplo, en países como Estados Unidos de América la deserción alcanza el $35 \%$, en tanto que en Italia el abandono de las carreras bordea el $60 \%$, y en España supera el 20\%. Por otra parte, la deserción universitaria en el Perú promedia una tasa del $17 \%$. Esto supone que de 40 a 50 mil jóvenes abandonan anualmente sus estudios universitarios, correspondiendo el $70 \%$ a estudiantes de universidades privadas y el $30 \%$ a estudiantes de universidades estatales (Mori, 2012), con las consiguientes pérdidas económicas de los padres de familia. De acuerdo con la investigación del Portal LOGROS (Plascencia: 2011): las pérdidas por la deserción universitaria en el año 2004 fueron de 99.23 millones de dólares. Mientras que durante el año 2005 se perdieron más de 100 millones de dólares. En ese año 42 mil alumnos abandonaron sus estudios, y la estimación es que al presente esta tendencia supere los 70 mil no graduados.

Por lo que se ve, entonces, el problema del fracaso académico tiene una trascendencia más allá de lo educativo y su alcance tiene efectos directos múltiples: por un lado en la salud psicológica del individuo y de la comunidad a la que pertenece y, por otro más amplio, en el desarrollo social y económico del país. En lo que respecta a la competencia psicológica es importante que, desde una visión de promoción de la salud biopsicosocial como la sostenida por Engel (ver Amigo, 2015: pp. 21-22), se identifiquen aquellos factores que mejor inciden en la adaptación de un joven a su vida universitaria y el alcance de sus objetivos en su formación profesional. Más aun cuando en nuestro país es muy difícil encontrar publicaciones de estudios que intenten evaluar estos aspectos, dado que la mayoría de investigaciones centran su atención únicamente en variables de riesgo asociadas a la conducta sexual o agresiva, y al abuso en el consumo de sustancias adictivas.

\footnotetext{
2. Así, corno dicen Neipp Lledó y Pons (2012), se puede considerar que los conceptos de calidad de vida, bienestar y ajuste se refieren a las valoraciones que los individuos realizan sobre el grado de satisfacción, capacidad y habilidades comparándolo con el modelo posible o ideal que han aprendido a usar de "medida".
} 
Carlos Velázquez, William Montgomery, Alejandro Dioses, Ricardo Pomalaya, Kevin Egúsquiza, Victoria Cavero, Angelika Pizarro, Ofelia Borja, David Duymovich, Giuliana Meza, Dongas Vázquez, Natalia Velázquez y Susana Cabrera

Lo que se pone de manifiesto de manera cierta en el campo de la salud es la evidencia de que, de una manera general, en la investigación se continúa enfatizando la ausencia de enfermedad. En este contexto, la psicología por el momento está interesada básicamente en la enfermedad, y no ha realizado muchos esfuerzos para clarificar las variables que determinan la salud mental de la población sana (Lluch; 1999; Taylor, 2003). Eso relieva la importancia de alcanzar una visión positiva sobre el problema de la salud (Seligman, 2008; Bernabé, Martín-Aragón, Lisbona y Tirado, 2012). Este modelo de análisis de salud biopsicosocial se relaciona, además con el de bienestar procedente de Carol Ryff (Diener\&Suh, 2000). "que promueve el dominio del entorno. el crecimiento personal, los buenos propósitos en la vida, la autonomía, la libre aceptación y las relaciones positivas con otros" (Luoke e Izquierdo, 2008; p. 103).

El ajuste o adaptación académica ha sido evaluado usando como indicadores el rendimiento escolar, la permanencia y la satisfacción reportada por los jóvenes (Neipp, Lledó \& Pons, 2012). Entre los factores subjetivos identificados como promotores de la adaptación respecto a diversas clases de situaciones estresantes se hayan la personalidad con una considerable habilidad social (Lazarus \& Folkman, 1986; Martin, 2002; Caballo, 2005, Adgo, 2015); una buena percepción de eficacia personal (Bandura, 1992; Londoña, 2007);una historia previa de buen rendimiento académico, soporte social real y percibido (Pratt, Bowers, Terzjan \& Hunsberger. 2000); y, asimismo, el afrontamiento positivo o centrado en el problema(Lazarus \& Folkman, 1986; Martínez-Correa. Reyes del Paso: García-León y GonzálezJareño, 2006), y el optimismo (Seligman, 2008).

Algunos de estos factores son conceptualizados como "salud positiva" puesto que no sólo se han asociado a mejor afrontamiento ante situaciones vitales, sino, además, han sido incluidos como los factores protectores fundamentales al interior de un amplio rango de riesgos para la salud. El constructo de "salud mental positiva" de los individuos fue abordado por la Dra. Marie Jahoda en su monografía de 1958: Cerent Concepts of Positive Mental Health, al advertir que; en su momento, lo que se llamaba "salud mental" era un término ambiguo y poco accesible a su definición, y, por ello, su trabajo tenía corno meta práctica la descripción del concepto. Jahoda - cuyo modelo fue delineado cuando se constituyó la Joint Commission on Mental iliness and Health para emprender un estudio integral de la salud mental en los Estados Unidos- conceptúa dicho concepto desde una perspectiva individual, considerando que aun cuando el ambiente y la cultura influyen en la salud y en la enfermedad, no es adecuado hablar de "sociedades enfermas" o de "comunidades enfermas". En este sentido, la salud mental positiva debe estudiarse desde una perspectiva psicológica sin descuidar la influencia mutua entre los aspectos físicos y mentales del ser humano; en el entendido de que poseer una buena salud física no es una condición suficiente para tener 
una buena salud mental (Vásquez y Hérvas, (2008).Esto es coincidente con las pautas del modelo de tipo biopsicosocial que antes hemos mencionado (Taylor, 2003; Amigo, 2015).

Lluch (1999), igual que Vázquez y Hervás (2008), describen el modelo de salud mental positiva como transversal a los ámbitos de la prevención de enfermedades y de la promoción de la salud, pero enfocando de manera preferencial en el aprendizaje de recursos para llevar a buen puerto esos objetivos. Para ellos, la propuesta de Jahoda procura responder a la pregunta de cuáles son los componentes de la salud mental positiva articulada desde una perspectiva de múltiples criterios, los cuales son: 1) actitudes hacía sí mismo; 2) crecimiento y autoactualización; 3) integración; 4) autonomía; 5) percepción de la realidad; y 6) dominio del entorno.

La tabla 1, obtenida de Vásquez y Hérvas (2008; pp. 22-23) detalla los aspectos multidimensionales del enfoque.

Tabla 1

Características de la salud mental positiva según el modelo de Jahoda (1958)

\begin{tabular}{ll}
\hline CRITERIOS & DIMENSIONES \\
\hline 1. Actitudes hacía sí mismo & - Accesibilidad del yo a la conciencia. \\
& - Concordancia yo real-yo ideal. \\
& - Sentimientos hacia uno mismo (Autoestima). \\
& - Sentido de identidad. \\
& - Motivación general hacia la vida. \\
2. Crecimiento, desarrollo y & - Implicación en la vida. \\
autoactualización & - Equilibrio de las fuerzas psíquicas (equilibrio entre el ello, el \\
3. Integración & yo y el superyó o entre los fenómenos psíquicos conscientes, \\
& preconscientes e inconscientes). \\
& - Visión integral de la vida. \\
& - Resistencia al estrés. \\
& - Autorregulación. \\
& - Conducta independiente. \\
4. Autonomía & - Percepción no distorsionada. \\
& - Empatía o sensibilidad social. \\
5. Percepción de la realidad & - Capacidad de amar. \\
6. Control ambiental & - Adecuación en el amor, el trabajo y el juego. \\
& - Adecuación en las relaciones interpersonales. \\
& - Eficiencia en el manejo de demandas situacionales. \\
& - Capacidad de adaptación y ajuste. \\
& - Eficiencia en resolución de problemas. \\
\hline & \\
& \\
&
\end{tabular}

Al ser, como se ve, un afronte bastante completo de salud biopsicosocial, éste constituye un modelo a tener en cuenta para la evaluación de los estudiantes universitarios, a fin de poder predecir posibles deserciones académicas en el transcurso de su carrera. En este sentido, consideramos el trabajo de Lluch (1999) 
como interesante para proporcionar un instrumento capaz de reflejar la salud mental positiva en todos esos aspectos: la Escala para evaluar la Salud Mental Positiva, que, en su versión original, tiene un abundante número de ítems (176), los cuales pueden adaptarse a nuestra realidad de acuerdo a los resultados de la investigación; tanto más cuanto dicha escala se ha empleado muy poco.

El propósito de esta indagación también se vincula al concepto de "universidades saludables" (Arroyo y Rice, 2009), una corriente internacional en base a la que se han establecido hace algún tiempo políticas estatales de promoción a través de nuestro Ministerio de Salud (Alayo, Bambarén, Gueiler, Magill, Sueiro, Valdivieso y Vera, 2013); con el objeto de promover la salud y decrementar estados mórbidos en instituciones de educación superior. De acuerdo con este objeto, se puede esperar que la investigación sobre el estilo de vida de los estudiantes permita llevar a conclusiones determinantes respecto a dichas políticas en cuanto a prácticas de riesgo o de protección en jóvenes universitarios (Arrivillaga, Salazar y Correa, 2003).

Es por todas las razones señaladas que, en resumen, el presente estudio procura establecer cuáles son las características de la salud mental positiva de los alumnos del Centro Pre-Universitario de la Universidad Nacional Mayor de San Marcos (UNMSM).

\section{MÉTODO}

El presente estudio es un diseño no experimental de tipo correlacional. La población de estudiantes regulares matriculados en el Centro Pre-universitario de la UNMSM en el semestre 2016-I fue de 4,995 alumnos, seleccionando una muestra no probabilística intencional de 938, de los cuales 436 son del género masculino y 502 del género femenino. 426 de ellos provienen de instituciones escolares públicas y 512 de instituciones privadas, donde el $29.3 \%$ postula al Área de Ciencias de la Salud, el 2.2\% al Área de Humanidades, 33.9\% al Área de Ciencias Sociales, $15 \%$ al Área de Ingeniería y 1\% al Área de Ciencias Económico-Empresariales.

Se utilizó la Escala de Evaluación de la Salud Mental Positiva (EVSMP) de Luch (1999), la cual fue adaptada a nuestra realidad (Anexo $\mathrm{N}^{\circ} 1$ ) en una muestra de 300 estudiantes, manteniendo su misma estructura: Satisfacción Personal (8 Ítems), Actitud Prosocial (4 ítems), Autocontrol (5 ítems), Autonomía (5 Ítems), Resolución de Problemas y Auto actualización (9 ítems) y Habilidades de Relaciones Interpersonales (7 ítems). Con respecto a la validez esta fluctúa entre .268 y .649.y una confiabilidad entre .637 y .791 (Anexo 2). 


\section{RESULTADOS}

Tabla 2

Prueba de Normalidad de Kolmogorov Smirnov.

\begin{tabular}{|c|c|c|c|c|c|c|c|}
\hline & & $\begin{array}{l}\text { SAT } \\
\text { PER } \\
\end{array}$ & $\begin{array}{l}\text { ACT } \\
\text { PROS } \\
\end{array}$ & $\begin{array}{l}\text { AUTO } \\
\text { CONT } \\
\end{array}$ & $\begin{array}{l}\text { AUTO } \\
\text { NO } \\
\end{array}$ & $\begin{array}{l}\text { RES } \\
\text { PROB } \\
\text { AUTO }\end{array}$ & $\begin{array}{l}\text { HAB } \\
\text { INTERP }\end{array}$ \\
\hline \multicolumn{2}{|l|}{$\mathrm{N}$} & 938 & 938 & 938 & 938 & 938 & 937 \\
\hline \multirow{2}{*}{$\begin{array}{l}\text { Parámetros } \\
\text { normales }^{\mathrm{a}, \mathrm{b}}\end{array}$} & & 26.75 & 6.89 & 10.78 & 15.53 & 16.80 & 20.90 \\
\hline & $\begin{array}{l}\text { Desviación } \\
\text { estándar }\end{array}$ & 4.07 & 2.09 & 2.88 & 2.89 & 4.33 & 3.50 \\
\hline \multirow{3}{*}{$\begin{array}{l}\text { Máximas } \\
\text { diferencias } \\
\text { extremas }\end{array}$} & & .134 & .151 & .087 & .121 & .086 & .067 \\
\hline & Positivo & .098 & .151 & .063 & .066 & .086 & .058 \\
\hline & Negativo & -.134 & -.083 & -.087 & -.121 & -.042 & -.067 \\
\hline \multicolumn{2}{|c|}{ Estadístico de prueba } & .134 & .151 & .087 & .121 & .086 & .067 \\
\hline \multicolumn{2}{|c|}{ Sig. asintótica (bilateral) } &, $000^{\mathrm{c}}$ &, $000^{c}$ &, $000^{\mathrm{c}}$ &, $000^{\mathrm{c}}$ &, $000^{\mathrm{c}}$ &, $000^{\mathrm{c}}$ \\
\hline
\end{tabular}

La Tabla 2, la Prueba de Normalidad, determina la utilización de estadística no paramétrica.

De otro lado la Tabla 3 muestra que existen diferencias en función al sexo en los factores Actitud Prosocial, siendo mayor en el sexo masculino, y en los factores Autocontrol, Resolución de Problemas-autoactualización y Habilidades de Relaciones Interpersonales siendo mayor en el sexo femenino.

En la Tabla 4, se observa que el turno en que estudian los alumnos muestran diferencias en los factores Satisfacción Personal y Autonomía es mayor en los alumnos del Turno Mañana y en los factores Actitud Personal y Resolución de Problemas-Autoactualización es mayor en el turno tarde.

Tabla 3

Prueba de Mann Whitney para determinar las diferencias en función al Sexo

SATPER ACTPROSOC AUTOCONT AUTONOMI RESPROAUTO HABINTERP

\begin{tabular}{lcccccc}
\hline $\begin{array}{l}\text { U de Mann- } \\
\text { Whitney }\end{array}$ & 103657.500 & 84086.000 & 95407.500 & 103808.000 & 99467.000 & 93458.000 \\
$\begin{array}{l}\text { W de } \\
\text { Wilcoxon }\end{array}$ & 229408.500 & 209837.000 & 190237.500 & 229559.000 & 194297.000 & 188288.000 \\
$Z$ & -1.292 & -6.100 & -3.306 & -1.259 & -2.309 & -3.727 \\
$\begin{array}{l}\text { Sig. asintótica } \\
\text { (bilateral) }\end{array}$ & .196 & .000 & .001 & .208 & .021 & .000 \\
\hline
\end{tabular}

a. Variable de agrupación: SEXO 
Carlos Velázquez, William Montgomery, Alejandro Dioses, Ricardo Pomalaya, Kevin Egúsquiza, Victoria Cavero, Angelika Pizarro, Ofelia Borja, David Duymovich, Giuliana Meza, Dongas Vázquez, Natalia Velázquez y Susana Cabrera

Tabla 4

Prueba de Mann Whitney para determinar las diferencias en función al Turno de Estudio

SATPER ACTPROSOC AUTOCONT AUTONOMI RESPROAUTO HABINTERP

\begin{tabular}{lcccccc}
\hline $\begin{array}{l}\text { U de Mann- } \\
\text { Whitney }\end{array}$ & 65702.000 & 70497.500 & 79671.500 & 72811.000 & 69948.500 & 73719.000 \\
$\begin{array}{l}\text { W de } \\
\text { Wilcoxon }\end{array}$ & 90902.000 & 325752.500 & 334926.500 & 98011.000 & 325203.500 & 98919.000 \\
$Z$ & -4.049 & -2.707 & -.084 & -2.037 & -2.839 & -1.743 \\
$\begin{array}{l}\text { Sig. asintótica } \\
\text { (bilateral) }\end{array}$ & .000 & .007 & .933 & .042 & .005 & .081 \\
\hline
\end{tabular}

En la Tabla 5, se observa que las Institución Educativas de donde proceden los alumnos no determina diferencias ente IE Publicas y Privadas.

En la Tabla 6, podemos observar que existen diferencias en función al Área Profesional a la que postulan los alumnos, solo en los factores Satisfacción Personal, y Autocontrol el Área de Ciencias Sociales y en el Factor Autonomía el Área de Humanidades.

En la Tabla $\mathrm{N}^{\circ}$, se muestra la no existencia de diferencias en función a la edad entre Adolescentes (de 14 a 18), Jóvenes (de 19 a 21 años) y Adultos-Jóvenes (de 22 a 39 años).

Tabla 5

Prueba de Mann Whitney para determinar las diferencias en función a la Institución Educativa de Procedencia

\begin{tabular}{|c|c|c|c|c|c|c|}
\hline & SATPER & ACTPROSOC & AUTOCONT & AUTONOMI & RESPROAUTO & HABINTERP \\
\hline $\begin{array}{l}\text { U de Mann- } \\
\text { Whitney }\end{array}$ & 104519.000 & 105513.500 & 106406.500 & 108305.500 & 104380.000 & 103937.000 \\
\hline $\begin{array}{l}\text { W de } \\
\text { Wilcoxon }\end{array}$ & 194619.000 & 195613.500 & 237222.500 & 198405.500 & 194480.000 & 193613.000 \\
\hline Z & -.931 & -.693 & -.471 & -.006 & -.964 & -1.012 \\
\hline $\begin{array}{l}\text { Sig. asintótica } \\
\text { (bilateral) }\end{array}$ & .352 & .488 & .638 & .995 & .335 & .311 \\
\hline
\end{tabular}

a. Variable de agrupación: IE

Tabla 6

Prueba de Mann Whitney para determinar las diferencias en función al Área Profesional a la que postulan

\begin{tabular}{lcccccc}
\hline & SATPER & ACTPROSOC & AUTOCONT & AUTONOMI & RESPROAUTO & HABINTERP \\
\hline $\begin{array}{l}\text { Chi- } \\
\text { cuadrado } \\
\text { gl }\end{array}$ & 11.589 & 9.074 & 9.561 & 9.685 & 9.398 & 2.766 \\
$\begin{array}{l}\text { Sig. } \\
\text { asintótica }\end{array}$ & .021 & 4 & 4 & 4 & 4 & 4 \\
\hline
\end{tabular}

a. Prueba de Kruskal Wallis b. Variable de agrupación: AREA 
Tabla 7

Prueba de Kruskal Wallis para determinar las diferencias en función a la Edad

\begin{tabular}{lrrrrrrr} 
& SATPER & ACTPROSOC & AUTOCONT & AUTONOMI & RESPROAUTO & HABINTERP \\
\hline $\begin{array}{l}\text { Chi- } \\
\text { cuadrado }\end{array}$ & .734 & 2.333 & 1.351 & 1.377 & 1.140 & .948 \\
\hline gl & 2 & 2 & 2 & 2 & 2 & 2 \\
\hline $\begin{array}{l}\text { Sig. } \\
\text { asintótica }\end{array}$ & .693 & .311 & .509 & .502 & .566 & .623 \\
\hline
\end{tabular}

En la Tabla 8, en cuanto a la correlación entre los factores de la EESMP, podemos observar que estas son significativas, en cuanto a la fuerza esta fluctúan entre bajas y moderadas y en cuatro a la dirección estas se expresan e Negativas entre la Satisfacción Personal con la Actividad Prosocial y la Resolución de Problemas-Autoactualización; entre la Actitud Prosocial con la Autonomía y la Habilidades de Relaciones Interpersonales; entre el Autocontrol con la Autonomía y las Habilidades de Relaciones Interpersonales y entre la Resolución de Problemas-Autoactualización con las Habilidades de Resolución interpersonal. En cuanto a la relación positiva entre el factor Satisfacción Personal con la autonomía y las Habilidades de Relaciones Interpersonal; entre la Actitud Prosocial y el Autocontrol y la Resolución de Problemas. Autoactualización; entre el Autocontrol y la Resolución de Problemas-Autoactualización y entre la Autonomía y la Habilidades de Relaciones interpersonal.

Tabla 8

Prueba Correlación No paramétrica de los Factores de la EESMP

\begin{tabular}{|c|c|c|c|c|c|c|c|}
\hline & & $\begin{array}{l}\text { SAT } \\
\text { PER }\end{array}$ & $\begin{array}{c}\text { ACT } \\
\text { PROS }\end{array}$ & $\begin{array}{l}\text { AUTO } \\
\text { CONT }\end{array}$ & $\begin{array}{l}\text { AUTO } \\
\text { NOMI }\end{array}$ & $\begin{array}{c}\text { RES PRO } \\
\text { AUTO }\end{array}$ & $\begin{array}{c}\text { HAB } \\
\text { INTERP }\end{array}$ \\
\hline SATPER & $\begin{array}{l}\mathrm{r} \\
\text { Sig. (bilateral) }\end{array}$ & 1.000 & $\begin{array}{r}-, 242^{\prime \prime} \\
.000\end{array}$ & $\begin{array}{r}-, 404^{* \prime \prime} \\
.000\end{array}$ & $\begin{array}{l}, 499^{n *} \\
.000\end{array}$ & $\begin{array}{c}-, 511^{N m} \\
.000\end{array}$ & $\begin{array}{l}, 455^{* m} \\
.000\end{array}$ \\
\hline ACTPROSOC & $\begin{array}{l}\mathrm{r} \\
\text { Sig. (bilateral) }\end{array}$ & & 1.000 & $\begin{array}{l}, 210^{* * *} \\
.000\end{array}$ & $\begin{array}{c}-, 153^{* *} \\
.000\end{array}$ & $\begin{array}{l}, 406^{* * *} \\
.000\end{array}$ & $\begin{array}{c}-, 489^{* *} \\
.000\end{array}$ \\
\hline AUTOCONT & $\begin{array}{l}\mathrm{r} \\
\text { Sig. (bilateral) }\end{array}$ & & & 1.000 & $\begin{array}{c}-, 329^{* *} \\
.000\end{array}$ & $\begin{array}{l}, 538^{* *} \\
.000\end{array}$ & $\begin{array}{l}-, 305^{* *} \\
.000\end{array}$ \\
\hline AUTONOMI & $\begin{array}{l}\mathrm{r} \\
\text { Sig. (bilateral) }\end{array}$ & & & & 1.000 & $\begin{array}{l}-, 369^{* *} \\
.000\end{array}$ & $\begin{array}{l}, 304^{* * *} \\
.000\end{array}$ \\
\hline RESPROAUTO & $\begin{array}{l}\mathrm{r} \\
\mathrm{Sig} . \text { (bilateral) } \\
\mathbf{N}\end{array}$ & & & & & 1.000 & $\begin{array}{c}-, 476^{* *} \\
.000\end{array}$ \\
\hline
\end{tabular}

\section{DISCUSIÓN}

La adaptación de la escala para nuestra realidad invirtió la polaridad de los siguientes ítems, el ítem 04 del Factor 1; el ítem 03 del Factor 2; el ítem 02 del Factor 3 y los ítems 11, 18 y20 del Factor 6.La correlación de los factores de la escala al igual que la versión original son todas estadísticamente significativas. 
Carlos Velázquez, William Montgomery, Alejandro Dioses, Ricardo Pomalaya, Kevin Egúsquiza, Victoria Cavero, Angelika Pizarro, Ofelia Borja, David Duymovich, Giuliana Meza, Dongas Vázquez, Natalia Velázquez y Susana Cabrera

En la correlación de la escala original se observan relaciones positivas, mientras que la adaptación para nuestra realidad muestra relaciones inversas entre los F1 con el F2, F3 y F5; entre el F2 con el F4 y F6; entre el F3 con los F4 y F6; entre el F4 con el F5 y entre el F5 con F6.

\section{CONCLUSIONES}

Los resultados estadísticos son satisfactorios en todos los factores reseñados, con lo cual podemos contar con un nuevo instrumento de medición de la variable "salud mental positiva" en nuestra realidad, que le permita a los profesionales de la educación realizar programas de prevención e intervención.

\section{REFERENCIAS}

Alayo, M., Bambarén, E., Gueiler, M., Magill, F., Sueiro, R., Valdivieso, M. y Vera, E. (2013). Guía universidades saludables. Lima: Comisión de Comunidades Saludables del Consorcio de Universidades.

Amigo, I. (2015). Manual de psicología de la salud. Madrid: Pirámide.

Arrivillaga, M. Salazar, I. y Correa, D. (2003). Creencias sobre la salud y su relación con las prácticas de riesgo o de protección en jóvenes universitarios. Colombia Médica, 34(4) 186-195.

Arroyo, H. y Rice, M. (Eds.) (2009).Una nueva mirada al movimiento de universidades promotoras de la salud en las Américas. Documento de trabajo desarrollado para el IV Congreso Internacional de Universidades Promotoras de la Salud. Puerto Rico: Organización Panamericana de la Salud.

Bandura, A. (1992). A self-efficaymechanism in psycholbiologicfunctioning. En: R. Schwarzer (Ed.): Self-efficacy: thougt control of action. Washington USA: Hemisphere.

Bernabé, M., Martín-Aragón, M., Lisbona, A. M. y Tirado, S. (2012). Aproximación a la psicología positiva, En M. del C. Terol, Y. Quiles y M. V. Pérez (Coords.). Manual de evaluación psicosocial en contextos de salud (pp. 164-197). Madrid: Pirámide.

Caballo, V. (2005). Manual de evaluacion y entrenamiento de las habilidades sociales. Madrid: Siglo XXI.

Diener, E. \& Suh, E. (Eds) (2000). Culture and Subjective well-being. Cambridge: Massachusetts Institute of Technology.

Henao, A. M. y Mahecha, J. C. (2005). Ajuste psicológico según edad y género en niños y jóvenes de estrato socioeconómico 1 y 2, de Bogotá. Suma Psicológica, 12(2), 197212.

Lazarus, R. \&Folkman, S. (1986). Estrés y procesos cognitivos. Barcelona España: Martínez Roca.

Londoño, C. (2007). Construcción de un modelo cognitivo social integrado para la prevención del abuso de alcohol en universitarios bogotanos. Tesis de maestría, Facultad de Psicología, Universidad Nacional de Colombia. 
Lucke, H. e Izquierdo, A. (2008). Terapia conductual. En Castanedo, C. (Ed.). Seis enfoques terapéuticos (pp. 53-104). México: El Manual Moderno.

Lluch, M. T. (1999). Construcción de una escala para evaluar la salud mental positiva. Tesis doctoral: Universidad de Barcelona.

Marín, M., Infante, E. y Troyano, Y. (2000). El fracaso académico en la universidad: Aspectos motivacionales e intereses profesionales. Revista Latinoamericana de Psicología, 32(3), 505-517.

Martin, N. (2002). Feelingoptimistic? Psychologist. 15 (6), 309-321.

Martínez-Correa, A., Reyes del paso, G., García-León, A. y González-Jareño, M. (2006). Optimismo / pesimismo disposicional y estrategias de afrontamiento del estrés. Psicothema, 18 (1) 66-77.

Mori, M. del P. (2012). Deserción universitaria en estudiantes de una universidad privada de Iquitos. Revista Digital de Investigación en Docencia Universitaria, 6(1), 60-83.

Neipp M. del C., Lledó, A. y Pons, N. (2012). Ajuste y adaptación psicosocial. En M. del C. Terol, Y. Quiles y M. V. Pérez (Coords.). Manual de evaluación psicosocial en contextos de salud (pp. 85-133). Madrid: Pirámide.

Plascencia, R. (2011). Deserción universitaria preocupa al mundo. Recuperado de http:// www.logrosperu.com/noticias/actualidad/707-mas-de-200-millones-de-dolares-seperdieron-en-dos-anos-por-desercion-universitaria.html

Pratt, M., Bowers, C., Terzjan, B. \& Hunsberger, B. (2000). To facilitate the transition to the university: evaluation of a so- cial program of intervention of and discussion. Journal of College Student Development, 41(4), 427-435.

OECD (2016). Education at a Glance. Recuperado de: http://www.oecd.org/edu/educationat-a-glance-19991487.htm

Ramírez, G. (2009, del 15 al 28 de junio). Deserción universitaria en la UNMSM. San Marcos al Día, 193(5). Recuperado de: http://www.unmsm.edu.pe/sanmarcosaldia/ index.php

Seligman, M. (2008). Positive health. Applied psychology, 57(1), 3-18.

Taylor, S. E. (2003). Psicología de la salud. México: McGraw-Hill.

Vázquez, C. y Hervás, C. (2008). Salud mental positiva: del síntoma al bienestar. En Psicología Positiva aplicada (pp. 17-39). Bilbao: Desclee de Brower. 
ANEXO N 1 EVSMP

DATOS GENERALES:

(Contesta o marca con una $X$ )

EDAD: .......... SEXO: (Masculino) (Femenino) TURNO: (Mañana) (Tarde)

CONDICIÓN DE LA INSTITUCIÓN EDUCATIVA DE PROCEDENCIA:

(Nacional) (Privada)

PROFESIÓN A LA QUE POSTULA:

\section{INSTRUCCIONES:}

Hola. En primer lugar, darte las gracias anticipadamente por tu colaboración. El cuestionario que te presentamos contiene una serie de afirmaciones referidas a la forma de pensar, sentir y hacer que son más o menos frecuentes en cada uno de nosotros. Para contestarlo lea cada frase y completa mentalmente el espacio de puntos suspensivos con la frecuencia que mejor se adapte a ti. A continuación, señala con una $(X)$ en las casillas de la derecha de tu respuesta.

Las posibles respuestas son:

(1) SIEMPRE O CASISIEMPRE

(2) A MENUDO O CON BASTANTE FRECUENCIA

(3) ALGUNAS VECES

\section{(4) NUNCA O CASI NUNCA}

No necesitas reflexionar mucho para contestar, ya que no hay respuestas correctas ni incorrectas. Lo que interesa es tu opinión. Tampoco trates de buscar la respuesta que quizá podría causar una "mejor impresión" ya que puedes estar segura que trataré la información con absoluta confidencialidad y anonimato. 


\section{POR FAVOR, CONTESTAA TODAS LAS AFIRMACIONES. MUCHAS GRACIAS.}

\begin{tabular}{|c|c|c|c|c|c|}
\hline & & $\begin{array}{c}\text { Siempre o } \\
\text { casi siempre }\end{array}$ & $\begin{array}{l}\text { A menudo o } \\
\text { con bastante } \\
\text { frecuencia }\end{array}$ & $\begin{array}{l}\text { Algunas } \\
\text { veces }\end{array}$ & $\begin{array}{c}\text { Nunca o casi } \\
\text { nunca }\end{array}$ \\
\hline 1 & $\begin{array}{l}\text { A mi, ... me resulta especialmente difícil aceptar a } \\
\text { los otros cuando tienen actitudes distintas a las mías }\end{array}$ & 1 & 2 & 3 & 4 \\
\hline 2 & Los problemas .... me bloquean fácilmente & 1 & 2 & 3 & 4 \\
\hline 3 & $\begin{array}{l}\text { A mi, .... me resulta especialmente difícil escuchar a } \\
\text { las personas que me cuentan sus problemas }\end{array}$ & 1 & 2 & 3 & 4 \\
\hline 4 & .... me gusto como soy & 1 & 2 & 3 & 4 \\
\hline 5 & $\begin{array}{l}\text {... soy capaz de controlarme cuando experimento } \\
\text { emociones negativas }\end{array}$ & 1 & 2 & 3 & 4 \\
\hline 6 & .... me siento a punto de explotar & 1 & 2 & 3 & 4 \\
\hline 7 & Para mi, la vida es .... aburrida y monótona & 1 & 2 & 3 & 4 \\
\hline 8 & $\begin{array}{l}\text { A mí, .... me resulta especialmente difícil dar apoyo } \\
\text { emocional }\end{array}$ & 1 & 2 & 3 & 4 \\
\hline 9 & $\begin{array}{l}\text {.... tengo dificultades para establecer relaciones } \\
\text { interpersonales profundas y satisfactorias con } \\
\text { algunas personas }\end{array}$ & 1 & 2 & 3 & 4 \\
\hline 10 & $\begin{array}{l}\text {... me preocupa mucho lo que los demás piensan } \\
\text { de mi }\end{array}$ & 1 & 2 & 3 & 4 \\
\hline 11 & $\begin{array}{l}\text { Creo que .... tengo mucha capacidad para ponerme } \\
\text { en el lugar de los demás y comprender sus } \\
\text { respuestas }\end{array}$ & 1 & 2 & 3 & 4 \\
\hline 12 & .... veo mi futuro con pesimismo & 1 & 2 & 3 & 4 \\
\hline 13 & $\begin{array}{l}\text { Las opiniones de los demás .... me influyen mucho a } \\
\text { la hora de tomar mis decisiones }\end{array}$ & 1 & 2 & 3 & 4 \\
\hline 14 & $\begin{array}{l}\text {.... me considero una persona menos importante } \\
\text { que el resto de personas que me rodean }\end{array}$ & 1 & 2 & 3 & 4 \\
\hline 15 & .... soy capaz de tomar decisiones por mi misma/o & 1 & 2 & 3 & 4 \\
\hline 16 & $\begin{array}{l}\text {.... intento sacar los aspectos positivos de las cosas } \\
\text { malas que me suceden }\end{array}$ & 1 & 2 & 3 & 4 \\
\hline 17 & .... intento mejorar como persona & 1 & 2 & 3 & 4 \\
\hline 18 & .... me considero "un/a buen/a alumno/a" & 1 & 2 & 3 & 4 \\
\hline 19 & .... me preocupa que la gente me critique & 1 & 2 & 3 & 4 \\
\hline 20 & .... creo que soy una persona sociable & 1 & 2 & 3 & 4 \\
\hline 21 & $\begin{array}{l}\text {.... soy capaz de controlarme cuando tengo } \\
\text { pensamientos negativos }\end{array}$ & 1 & 2 & 3 & 4 \\
\hline
\end{tabular}


Carlos Velázquez, William Montgomery, Alejandro Dioses, Ricardo Pomalaya, Kevin Egúsquiza, Victoria Cavero, Angelika Pizarro, Ofelia Borja, David Duymovich, Giuliana Meza, Dongas Vázquez, Natalia Velázquez y Susana Cabrera

\begin{tabular}{|c|c|c|c|c|c|}
\hline 22 & $\begin{array}{l}\text {.... soy capaz de mantener un buen nivel de } \\
\text { autocontrol en las situaciones conflictivas de mi vida }\end{array}$ & 1 & 2 & 3 & 4 \\
\hline 23 & .... pienso que soy una persona digna de confianza & 1 & 2 & 3 & 4 \\
\hline 24 & $\begin{array}{l}\text { A mi, .... me resulta especialmente difícil entender } \\
\text { los sentimientos de los demás }\end{array}$ & 1 & 2 & 3 & 4 \\
\hline 25 & .... pienso en las necesidades de los demás & 1 & 2 & 3 & 4 \\
\hline 26 & $\begin{array}{l}\text { Si estoy viviendo presiones exteriores desfavorables } \\
\text {... soy capaz de continuar manteniendo mi equilibrio } \\
\text { personal }\end{array}$ & 1 & 2 & 3 & 4 \\
\hline 27 & $\begin{array}{l}\text { Cuando hay cambios en mi entorno .... intento } \\
\text { adaptarme }\end{array}$ & 1 & 2 & 3 & 4 \\
\hline 28 & $\begin{array}{l}\text { Delante de un problema ....soy capaz de solicitar } \\
\text { información. }\end{array}$ & 1 & 2 & 3 & 4 \\
\hline 29 & $\begin{array}{l}\text { Los cambios que ocurren en mi rutina habitual.... me } \\
\text { estimulan. }\end{array}$ & 1 & 2 & 3 & 4 \\
\hline 30 & $\begin{array}{l}\text {... tengo dificultades para relacionarme } \\
\text { abiertamente con mis profesores/jefes }\end{array}$ & 1 & 2 & 3 & 4 \\
\hline 31 & .... creo que soy un/a inútil y no sirvo para nada & 1 & 2 & 3 & 4 \\
\hline 32 & $\begin{array}{l}\text {.... trato de desarrollar y potenciar mis buenas } \\
\text { aptitudes }\end{array}$ & 1 & 2 & 3 & 4 \\
\hline 33 & .... me resulta difícil tener opiniones personales & 1 & 2 & 3 & 4 \\
\hline 34 & $\begin{array}{l}\text { Cuando tengo que tomar decisiones importantes .... } \\
\text { me siento muy insegura/o }\end{array}$ & 1 & 2 & 3 & 4 \\
\hline 35 & .... soy capaz de decir no cuando quiero decir no & 1 & 2 & 3 & 4 \\
\hline 36 & $\begin{array}{l}\text { Cuando se me plantea un problema .... intento } \\
\text { buscar posibles soluciones }\end{array}$ & 1 & 2 & 3 & 4 \\
\hline 37 & .... me gusta ayudar a los demás & 1 & 2 & 3 & 4 \\
\hline 38 & .... me siento insatisfecha/o conmigo misma/o & 1 & 2 & 3 & 4 \\
\hline 39 & .... me siento insatisfecha/o de mi aspecto físico & 1 & 2 & 3 & 4 \\
\hline
\end{tabular}




\section{ANEXO No 2}

Validez de la EVSMP a través del Método del Ítem Test

SATISFACCION PERSONAL

\begin{tabular}{cl}
\hline İtems & $\mathrm{r}$ \\
\hline 4 & .495 \\
6 & .401 \\
7 & .407 \\
12 & .503 \\
14 & .560 \\
31 & .552 \\
38 & .524 \\
39 & .529 \\
\hline
\end{tabular}

AUTOCONTROL

\begin{tabular}{cc}
\hline Ítems & $\mathrm{r}$ \\
\hline 2 & .358 \\
5 & .575 \\
21 & .632 \\
22 & .649 \\
26 & .439 \\
\hline
\end{tabular}

AUTONOMIA

\begin{tabular}{cc}
\hline Ítems & $\mathrm{r}$ \\
\hline 10 & .518 \\
13 & .516 \\
19 & .530 \\
33 & .347 \\
34 & .492 \\
\hline
\end{tabular}

RESOLUCIÓN DE PROBLEMAS Y AUTOACTUALIZACION

\begin{tabular}{cc}
\hline İtems & $\mathrm{r}$ \\
\hline 15 & .439 \\
16 & .481 \\
17 & .464 \\
27 & .395 \\
28 & .374 \\
29 & .315 \\
32 & .429 \\
35 & .268 \\
36 & .582 \\
\hline
\end{tabular}

HABILIDADES DE RELACIONES INTERPERSONAL

\begin{tabular}{cc}
\hline items & $\mathrm{r}$ \\
\hline 8 & .410 \\
9 & .492 \\
11 & .311 \\
18 & .278 \\
20 & .522 \\
24 & .384 \\
30 & .358 \\
\hline
\end{tabular}

Confiabilidad a través del Alfa de Crombach

\begin{tabular}{cc}
\hline FACTORES & $\mathrm{r}$ \\
\hline SATISFACCION PERSONAL & .791 \\
ACTITUD PROSOCIAL & .627 \\
AUTOCONTROL & .756 \\
AUTONOMIA & .722 \\
RESOLUCION DE PROBLEMAS Y AUTOACTUALIZACION & .742 \\
HABILIDADES DE RELACIONES INTERPERSONALES & .683 \\
\hline
\end{tabular}


